\title{
Immunoscore-has it scored for colon cancer precision medicine?
}

\author{
Robert N. Jorissen ${ }^{1,2}$, Anuratha Sakthianandeswaren ${ }^{1,2}$, Oliver M. Sieber ${ }^{1,2,3,4}$ \\ ${ }^{1}$ Systems Biology and Personalised Medicine Division, The Walter and Eliza Hall Institute of Medical Research, Parkville, Victoria, Australia; \\ ${ }^{2}$ Department of Medical Biology, The University of Melbourne, Parkville, Victoria, Australia; ${ }^{3}$ Department of Surgery, The University of Melbourne, \\ Parkville, Victoria, Australia; ${ }^{4}$ Department of Biochemistry \& Molecular Biology, Monash University, Clayton, Victoria, Australia \\ Correspondence to: Oliver M. Sieber. Systems Biology and Personalised Medicine Division, The Walter and Eliza Hall Institute of Medical Research, \\ Parkville, Victoria, Australia. Email: sieber.o@wehi.edu.au. \\ Provenance: This is an invited Editorial commissioned by Section Editor Zhihua Zhou, (Department of Pathology, The 101 Hospital of People's \\ Liberation Army Wuxi, Wuxi, China). \\ Comment on: Pagès F, Mlecnik B, Marliot F, et al. International validation of the consensus Immunoscore for the classification of colon cancer: a \\ prognostic and accuracy study. Lancet 2018;391:2128-39.
}

Submitted Aug 23, 2018. Accepted for publication Sep 12, 2018.

doi: $10.21037 / \mathrm{atm} .2018 .09 .29$

View this article at: http://dx.doi.org/10.21037/atm.2018.09.29

For patients with early-stage resectable colon cancer, the principal method for determining prognosis is based on histopathological characterization of the tumor, including the depth of tumor penetration ( $\mathrm{T}$ stage) and spread to nearby lymph nodes ( $\mathrm{N}$ stage), with the American Joint Committee on Cancer (AJCC) staging system perhaps the most widely used risk classification system. Other tumor risk parameters considered in clinical practice include grade of differentiation, lymphovascular and perinuclear invasion, microsatellite instability (MSI) status, inadequately sampled lymph nodes, and obstruction or perforation of the bowel at presentation (1).

High levels of tumor-infiltrating lymphocytes have been associated with lower rates of recurrence and longer survival in patients with colon cancer in multiple studies (2-9). Studies of lymphocyte sub-populations have further related better outcomes to the host adaptive T-lymphocytic response including $\mathrm{CD} 3+, \mathrm{CD} 57+, \mathrm{CD} 8+, \mathrm{CD} 45 \mathrm{RO}+$ or FOXP3 + cells $(7,10,11)$. Despite strong evidence of prognostic value, the assessment of tumor-infiltrating lymphocytes has not entered routine clinical use due to a lack of endorsed scoring systems with robust validation.

To address this deficit, the international Immunoscore project $(12,13)$ was established to develop and evaluate a standardized approach for testing a set of consensus immune markers, with results recently presented by Pagès and colleagues in The Lancet (14). A consortium of 14 centers in 13 countries from North America, Europe and
Asia assessed a predefined Immunoscore assay in patients with stage I-III colon cancer, assigned to a training set (TS), an internal validation set (IVS), or an external validation set (EVS). The densities of CD3+ and CD8+ T cells were determined in the tumor center and invasive margin regions using digital image-analysis on immunostained sections. Measurements were assigned into percentiles based on the distribution of results observed in a reference population, defined as the combined TS and IVS cohorts, although use of information from the latter to some extent diminishes the independence of the IVS cohort in terms of the presented validation. The Immunoscore for each patient was calculated as the mean of the four percentiles obtained for $\mathrm{CD} 3+$ and $\mathrm{CD} 8+\mathrm{T}$ cell counts at either the tumor center or invasive margin. Immunoscores were categorized into three groups, with a mean percentile $0-25 \%, 25-70 \%$, $70-100 \%$ as Immunoscore-low, -intermediate and -high, respectively. The lower (25\%) and upper (70\%) thresholds were selected from the TS cohort data to generate balanced proportions of patients within each Immunoscore group, with approximately one quarter of samples in the both the low and high categories. However, no detailed assessment of the appropriateness of these thresholds for classification of outcomes was presented, and formal determination of optimal cut-offs may perhaps result in an improved identification of low- and high-risk individuals.

Out of 3,539 patients with processed samples, the Immunoscore assay was successfully performed for $90 \%$ 
of individuals, demonstrating broad applicability, with 357 patients excluded after biomarker quality control (missing CD3 + or CD8+ T cell counts at either tumor center or invasive margin, or low staining quality). A further 577 patients were excluded after clinical data quality control. Among the 2,681 patients included in the analyses, 700 were assigned to the TS, 636 to the IVS and 1,345 to the EVS cohorts. In a set of control slides distributed between centers, the Immunoscore assay yielded staining intensities within a $20 \%$ maximum deviation of the intensity from the adjacent slide stained by the reference center for $>80 \%$ of slides. While indicating a generally high staining homogeneity, it would be of interest to propagate this information with respect to prediction uncertainty for patient classification. Re-analysis of selected images from 36 tumors representing Immunoscores ranging from the $2.5^{\text {th }}$ to the $90^{\text {th }}$ percentile by eight pathologists from different centers demonstrated strong interobserver reproducibility ( $r=0.97$ for tumor center; $r=0.97$ for invasive margin). However, some departure from the line of equality was evident for Immunoscores determined by different observers, which might impact the consistency of classification of patients into prognostic groups (see data presented in article Figure 2D and Figure S6D).

In comparison, visual assessment of the density of tumor infiltrating $\mathrm{T}$ cells in a subset of 268 tumors stained with haematoxylin and eosin by 11 independent evaluators was reported to yield low reproducibility. Although this assessment was guided by a set of reference slides (three slides each for Immunoscore-high, -intermediate and -low groups), it remains unclear whether higher reproducibility could have been achieved with more detailed standardization of the scoring system as described by others or the use of digital image analysis $(9,15-18)$.

The results presented by Pagès et al. validate the consensus Immunoscore as a prognostic assay for recurrence-free survival (primary endpoint), disease-free survival and overall survival in patients with stage I-III colon cancer. In the TS, IVS and ES cohorts, risk of recurrence at 3 years was $5 \%, 6 \%$ and $14 \%$ for the Immunoscore-high, $17 \%, 10 \%$ and $24 \%$ for the Immunoscore-intermediate and $26 \%, 20 \%$ and $36 \%$ for the Immunoscore-low groups, respectively. In the Cox multivariable analysis stratified by center, the Immunoscore association with outcome was independent of patient age, gender, $\mathrm{T}$ stage, $\mathrm{N}$ stage and MSI. However, other available prognostic variables such as grade of differentiation, lymphovascular and perinuclear invasion were not included in the models and would have been of interest to consolidate the evidence for the independent prognostic value of the Immunoscore. In addition, adjuvant chemotherapy use was not considered which would appear to be a major omission. The Immunoscore was valid across North American, European and Asian populations when comparing Immunoscore-high versus -low groups for recurrence-free survival, although this was only presented for univariate analysis. Whether population-specific reference distributions could improve predictions remains to be fully explored.

Combining all cohorts, the Immunoscore had a similar predictive accuracy for overall survival as $\mathrm{T}$ and $\mathrm{N}$ stage when assessed as single biomarkers, as quantified by the integrated area under the ROC curve (iAUC) with 1,000x bootstrap resampling. Furthermore, the addition of Immunoscore to a model comprising all available prognostic variables (age, gender, $\mathrm{T}$ stage, $\mathrm{N}$ stage, MSI, mucinous histology, sidedness, venous emboli, lymphatic invasion, perineural invasion, differentiation) resulted in a statistically significant model improvement. However, the observed increase in iAUC was only from 0.60 to 0.62 , where 0.5 corresponds to random prediction and 1.0 to perfect prediction (these data are presented in article Figure 4A). The utility of the Immunoscore for individual patient predictions, even when combined with other variables, thus appears to remain limited. Corresponding results were not reported for recurrence-free and disease-free survival.

MSI was found to be strongly associated with a high Immunoscore (45\% in MSI vs. $21 \%$ in MSS cancers across all cohorts), consistent with previous findings $(9,19)$ and likely related to the higher neoantigen load in MSI tumors (20,21). In multivariable, stratified Cox models combining MSI status with the Immunoscore, MSI remained a significant factor for time to recurrence but was not a significant factor for disease-free and overall survival and was dependent on Immunoscore. Given the emerging use of immunotherapy agents in metastatic MSI colon cancer, exploration of the Immunoscore as a predictive marker for patients presenting with metastatic disease and undergoing resection of the primary tumor will be of high interest.

A subgroup analysis was presented for patients with stage II colon cancer, for whom adjuvant chemotherapy use is contentious and restricted to patients with high-risk features including T4 stage, inadequately sampled lymph nodes, high grade, lymphovascular or perineural invasion, and obstruction or perforation of the bowel at presentation $(1,22)$. Using the three-category Immunoscore, patients with stage II colon cancer and high Immunoscore had a 
3 -year risk of recurrence of $6 \%$, compared to $11 \%$ and $20 \%$ for patients with intermediate and low Immunoscore, respectively. The Immunoscore was significant in multivariable, stratified Cox models for recurrence-free, disease-free and overall survival, and was further reported to be prognostic for stage II patients with MSS cancers who were not receiving chemotherapy, although details for the latter comparison were not provided. These data suggest that the Immunoscore may be of utility for prediction of prognosis in low-risk stage II patients, although this was based on the combined cohorts (TS, IVS \& EVS) and findings were not discussed in the context of clinical decision making. The potential impact of using the Immunoscore for guiding adjuvant treatment allocation for patients with stage II colon cancer thus remains uncertain and will require further investigation.

In conclusion, the results presented by Pagès et al. establish reproducibility and robustness of the consensus Immunoscore assay in patients with stage I-III colon cancer and validate the Immunoscore as a promising prognostic factor. Prospective studies will now be required to test the value of the Immunoscore for guiding clinical decisions beyond the use of routine clinical and histopathological parameters. Perhaps the most relevant application is for directing adjuvant treatment decisions in stage II patients, while utility would appear limited given current routine practice for stage I (surgery only) or stage III disease (surgery plus adjuvant chemotherapy where possible). The clinical value of the Immunoscore also needs to be viewed in the context of other emerging technologies for identification of patients at high risk of recurrence such as molecular classification systems and liquid biopsy approaches $(23,24)$. A recent analysis of circulating tumor DNA (ctDNA) to detect minimal residual disease in patients with stage II colon cancer has demonstrated very high accuracy (24); perhaps the Immunoscore could play a role in identifying patients for ctDNA analyses. Some challenges remain with respect to the practical implementation of the consensus Immunoscore, including decreasing the number of patients for whom the Immunoscore cannot be measured, and definition of the baseline dataset against which Immunoscore percentiles will be scored. Given the reliance of the Immunoscore assay on a reference population for classification into three prognosis groups, monitoring of calibration between pathology providers will be a key concern. Identification of the reasons underlying the variation in tumor immune cell infiltrates, beyond neoantigen load, remains an active area of research. The tumor microenvironment, intestinal microbiota, patient genetics, comorbidities, diet and use of medications may play important roles. The development of a standardized Immunoscore assay opens new avenues for robust population-scale cancer immunology research, and if clinical utility and cost-effectiveness are proven, provides a template for implementation into clinical practice.

\section{Acknowledgements}

None.

\section{Footnote}

Conflicts of Interest: The authors have no conflicts of interest to declare.

\section{References}

1. Locker GY, Hamilton S, Harris J, et al. ASCO 2006 update of recommendations for the use of tumor markers in gastrointestinal cancer. J Clin Oncol 2006;24:5313-27.

2. Svennevig JL, Lunde OC, Holter J, et al. Lymphoid infiltration and prognosis in colorectal carcinoma. $\mathrm{Br} \mathrm{J}$ Cancer 1984;49:375-7.

3. Ropponen KM, Eskelinen MJ, Lipponen PK, et al. Prognostic value of tumour-infiltrating lymphocytes (TILs) in colorectal cancer. J Pathol 1997;182:318-24.

4. Klintrup K, Makinen JM, Kauppila S, et al. Inflammation and prognosis in colorectal cancer. Eur J Cancer 2005; $41: 2645-54$.

5. Galon J, Costes A, Sanchez-Cabo F, et al. Type, density, and location of immune cells within human colorectal tumors predict clinical outcome. Science 2006;313:1960-4.

6. Ogino S, Nosho K, Irahara N, et al. Lymphocytic reaction to colorectal cancer is associated with longer survival, independent of lymph node count, microsatellite instability, and $\mathrm{CpG}$ island methylator phenotype. Clin Cancer Res 2009;15:6412-20.

7. Roxburgh CS, McMillan DC. The role of the in situ local inflammatory response in predicting recurrence and survival in patients with primary operable colorectal cancer. Cancer Treat Rev 2012;38:451-66.

8. Mei Z, Liu Y, Liu C, et al. Tumour-infiltrating inflammation and prognosis in colorectal cancer: systematic review and meta-analysis. Br J Cancer 2014;110:1595-605.

9. Williams DS, Mouradov D, Jorissen RN, et al. 
Lymphocytic response to tumour and deficient DNA mismatch repair identify subtypes of stage II/III colorectal cancer associated with patient outcomes. Gut 2018. [Epub ahead of print].

10. Pages F, Kirilovsky A, Mlecnik B, et al. In situ cytotoxic and memory $\mathrm{T}$ cells predict outcome in patients with early-stage colorectal cancer. J Clin Oncol 2009;27:5944-51.

11. Liska V, Vycital O, Daum O, et al. Infiltration of colorectal carcinoma by S100+ dendritic cells and CD57+ lymphocytes as independent prognostic factors after radical surgical treatment. Anticancer Res 2012;32:2129-32.

12. Galon J, Pages F, Marincola FM, et al. Cancer classification using the Immunoscore: a worldwide task force. J Transl Med 2012;10:205.

13. Galon J, Mlecnik B, Bindea G, et al. Towards the introduction of the 'Immunoscore' in the classification of malignant tumours. J Pathol 2014;232:199-209.

14. Pagès $\mathrm{F}$, Mlecnik $\mathrm{B}$, Marliot $\mathrm{F}$, et al. International validation of the consensus Immunoscore for the classification of colon cancer: a prognostic and accuracy study. Lancet 2018;391:2128-39.

15. Rozek LS, Schmit SL, Greenson JK, et al. TumorInfiltrating Lymphocytes, Crohn's-Like Lymphoid Reaction, and Survival From Colorectal Cancer. J Natl Cancer Inst 2016;108.

16. Hendry S, Salgado R, Gevaert T, et al. Assessing TumorInfiltrating Lymphocytes in Solid Tumors: A Practical Review for Pathologists and Proposal for a Standardized Method from the International Immuno-Oncology Biomarkers Working Group: Part 2: TILs in Melanoma, Gastrointestinal Tract Carcinomas, Non-Small Cell Lung Carcinoma and Mesothelioma, Endometrial and Ovarian Carcinomas, Squamous Cell Carcinoma of the Head and

Cite this article as: Jorissen RN, Sakthianandeswaren A, Sieber OM. Immunoscore-has it scored for colon cancer precision medicine? Ann Transl Med 2018;6(Suppl 1):S23. doi: 10.21037/atm.2018.09.29
Neck, Genitourinary Carcinomas, and Primary Brain Tumors. Adv Anat Pathol 2017;24:311-35.

17. Iseki Y, Shibutani M, Maeda K, et al. A new method for evaluating tumor-infiltrating lymphocytes (TILs) in colorectal cancer using hematoxylin and eosin (H-E)stained tumor sections. PLoS One 2018;13:e0192744.

18. Eriksen AC, Andersen JB, Kristensson M, et al. Computerassisted stereology and automated image analysis for quantification of tumor infiltrating lymphocytes in colon cancer. Diagn Pathol 2017;12:65.

19. Mlecnik B, Bindea G, Angell HK, et al. Integrative Analyses of Colorectal Cancer Show Immunoscore Is a Stronger Predictor of Patient Survival Than Microsatellite Instability. Immunity 2016;44:698-711.

20. Schwitalle Y, Linnebacher M, Ripberger E, et al. Immunogenic peptides generated by frameshift mutations in DNA mismatch repair-deficient cancer cells. Cancer Immun 2004;4:14.

21. Williams DS, Bird MJ, Jorissen RN, et al. Nonsense mediated decay resistant mutations are a source of expressed mutant proteins in colon cancer cell lines with microsatellite instability. PLoS One 2010;5:e16012.

22. Benson AB 3rd, Schrag D, Somerfield MR, et al. American Society of Clinical Oncology recommendations on adjuvant chemotherapy for stage II colon cancer. J Clin Oncol 2004;22:3408-19.

23. Sinicrope FA, Shi Q, Smyrk TC, et al. Molecular markers identify subtypes of stage III colon cancer associated with patient outcomes. Gastroenterology 2015;148:88-99.

24. Tie J, Wang Y, Tomasetti C, et al. Circulating tumor DNA analysis detects minimal residual disease and predicts recurrence in patients with stage II colon cancer. Sci Transl Med 2016;8:346ra92. 